\title{
Trigeminal Neuralgia Due to Red Vein Draining a Supratentorial Arteriovenous Malformation: Case Report
}

\author{
Takuro Inoue ${ }^{1}$ Ayako Shima ${ }^{1}$ Hisao Hirai ${ }^{1}$ Fumio Suzuki ${ }^{1}$ Masayuki Matsuda ${ }^{1}$ \\ ${ }^{1}$ Department of Neurosurgery, Subarukai Kotoh Kinen Hospital, \\ Higashiohmi, Shiga, Japan \\ Address for correspondence Takuro Inoue, MD, PhD, Department of \\ Neurosurgery, Subarukai Kotoh Kinen Hospital, 2-1 Hiramatsu-cho, \\ Higashiohmi, Shiga 527-0134, Japan (e-mail: takuro39@gmail.com).
} J Neurol Surg Rep 2016;77:e109-e112.

\author{
Abstract \\ Keywords \\ - arteriovenous \\ malformation \\ - trigeminal neuralgia \\ - vein \\ - microvascular \\ decompression
}

Trigeminal neuralgia (TGN) is rarely caused by arteriovenous malformation (AVM). The AVMs causing TGN are reported mostly in the ipsilateral posterior fossa. The culprit vessels are dilated feeding artery or nidus itself. We present a rare case of TGN caused by dilated draining veins from a supratentorial AVM. The patient suffered from TGN with an incidentally found large AVM, which had been left untreated. The neuralgia was successfully relieved by microvascular decompression. Dilated red veins compressed the nerve at the root entry zone and distant cisternal portion of the nerve. Technically, transposition is not practical for fragile, dilated red veins with high pressure. Interposition is safer method in this case.

\section{Introduction}

Arteriovenous malformation (AVM)-related trigeminal neuralgia (TGN) is rare. The AVM is reported to be mostly located in the ipsilateral posterior fossa, and the cause of the TGN is compression on the trigeminal nerve by nidus itself or dilated feeding artery. ${ }^{1-4}$ We present here a rare case of TGN caused by red veins draining a large supratentorial AVM.

\section{Case Report}

A 60-year-old man presented with severe facial pain on the left side when eating or washing face. He was diagnosed with TGN and had been on carbamazepine for 15 years. His pain had become more severe and medically intractable since the past 1 year. He had a large diffuse supratentorial AVM located mainly in the right parietooccipital region, which had been observed for a long time because it was asymptomatic and considered to be difficult to treat ( - Fig. 1). The radiologically visible draining veins were mainly located supratentorially. Magnetic resonance imaging (MRI) revealed that the superior cerebellar artery courses away from the nerve, indicating it is unlike cause of the neuralgia. Contrast-enhanced study showed a vein compressing trigeminal nerve adjacent to the superior petrosal vein (-Fig. 2). Three-dimensional picture created by GammaPlan (ELEKTA, Stockholm, Sweden) clearly showed that lateral and medial pontomesencephalic veins merge at root entry zone and turn around the nerve, and then flow into superior petrosal sinus (- Fig. 3). There are no feeding arteries to the AVM around the nerve.

His TGN was so severe and intolerable that he asked for surgical intervention. The microvascular decompression (MVD) was undertaken via the retrosigmoid approach. During craniotomy, we noted the emissary vein was red in color and carried as high pressure as arterial blood. Intradurally, the superior petrosal vein was also red and pulsated like arteries, suggesting arterial blood flowing through the vein, that is, the so-called "red vein" (-Fig. 4A). The root entry zone of the trigeminal nerve was compressed by arterialized pontomesencephalic vein ( - Fig. 4B). The red vein winded around the nerve distally and then merged into the superior petrosal sinus just behind suprameatal tubercle (-Fig. $\mathbf{4 C}$ ). The veins were red in color and pulsated. It was apparent that arterial blood from the AVM was flowing through these veins. received

January 26, 2016

accepted

May 9, 2016
DOI http://dx.doi.org/

10.1055/s-0036-1584817. ISSN 2193-6358. (c) 2016 Georg Thieme Verlag KG
Stuttgart · New York

License terms

(1) $\Theta \circledast$ 

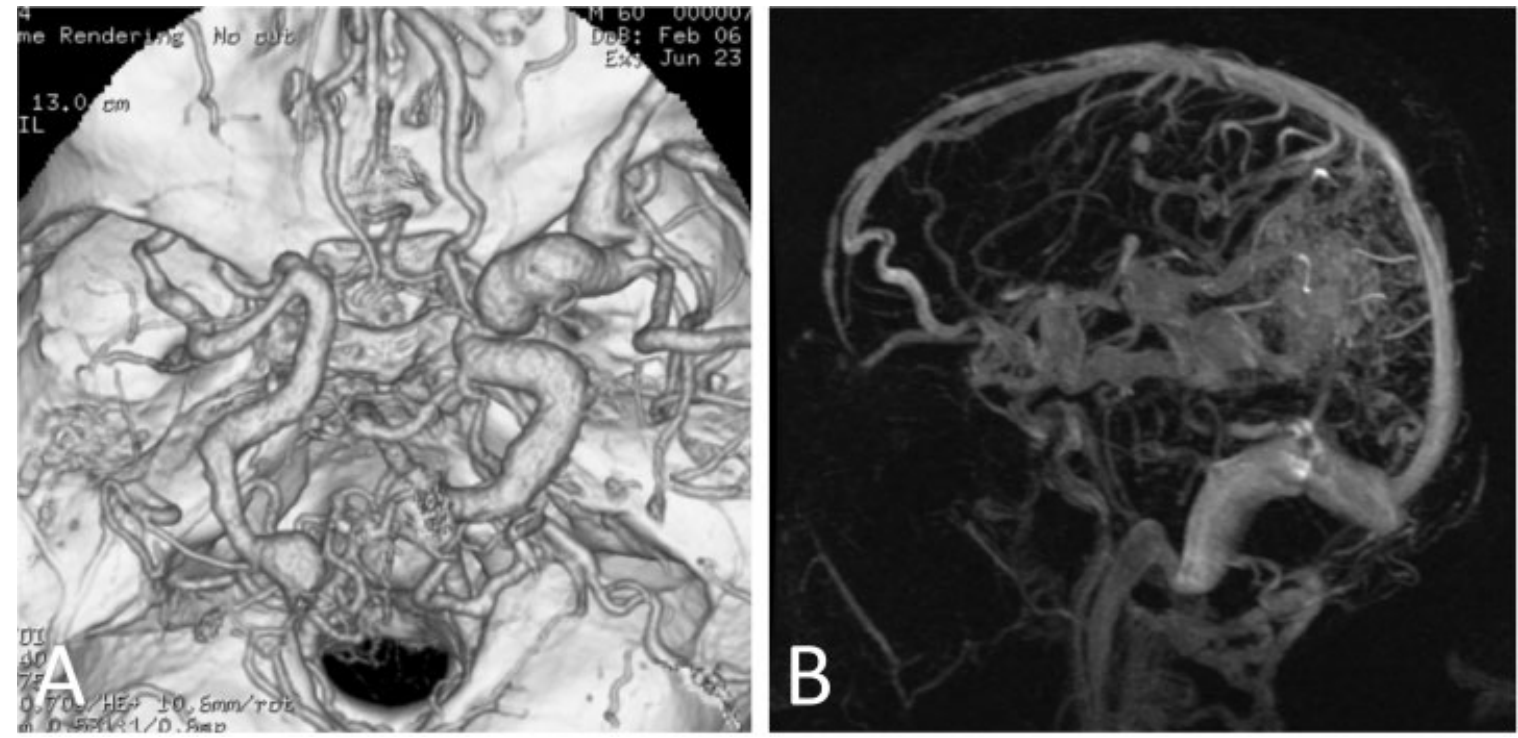

Fig.1 (A) Three-dimensional computed tomography shows a large arteriovenous malformation. (B) Magnetic resonance angiography shows that the nidus is located supratentorially.

Interposition of the vein was performed because the cutting of the vein might cause uncontrollable bleeding from the nidus of the AVM. As the vein did not have enough length or flexibility to be transposed, interposition by Teflon felt was performed. Superior cerebellar artery was coursing a bit above the nerve, suggesting negative relation to his neuralgia. His pain was relieved postoperatively without any noticeable complication. He was free from medication and had no recurrence at the final visit 6 months after the operation.

\section{Discussion}

AVM-related TGN is rare. It is reported that its incidence is 0 to $1.5 \%$ of the population who underwent MVD for TGN. ${ }^{4-6}$ In all the reported AVM-related TGN cases, the underlying AVM niduses locate in the posterior fossa. Trigeminal nerve may be compressed by the nidus itself or by a feeding artery of the AVM, such as superior cerebellar artery and basilar artery with flow-related dilation. ${ }^{1-4,7-9}$

Mori et $\mathrm{al}^{3}$ reviewed reported cases of TGN associated with AVM after MRI era and divided them into four groups according to the AVM location, such as intrinsic trigeminal nerve, cerebellopontine angle, distant and dural (petrotentorial). The AVMs are mostly located in the ipsilateral posterior fossa, except one case in which TGN was caused by a dilated draining red vein from the AVM located on the contralateral side..$^{10}$ Our case is considered to be rare as the AVM was located in the supratentorial region and dilated draining red veins were the cause of the neuralgia. The nidus was so large that the arterial blood massively flowed into the posterior fossa and formed dilated draining red veins around the trigeminal nerve.
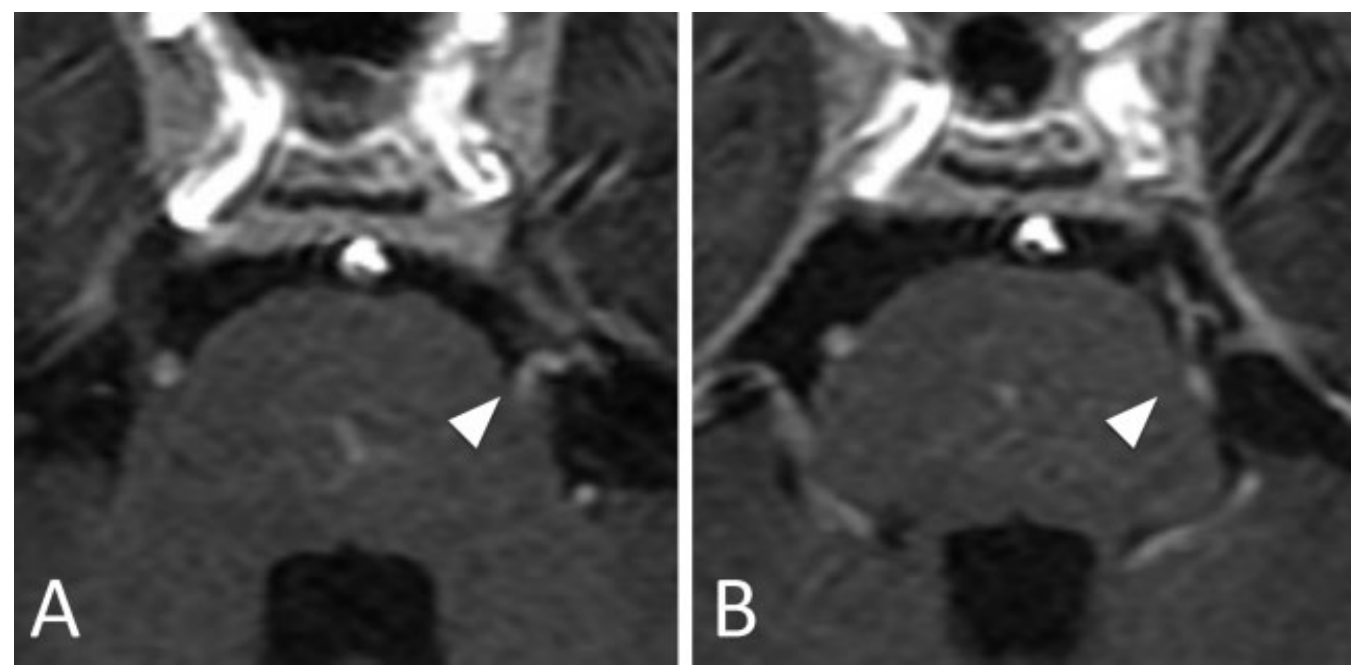

Fig. 2 (A,B) The contrast enhanced magnetic resonance imaging ( $T 1$ spoiled gradient recalled) reveals neurovascular compression on the trigeminal nerve (white arrow heads). 

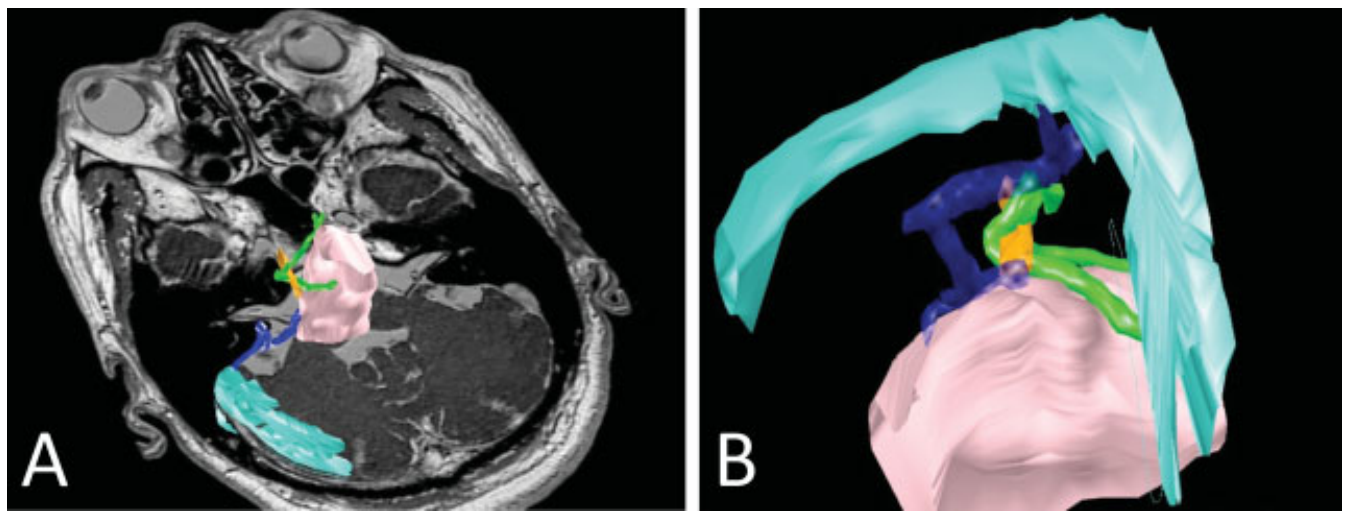

Fig. 3 (A) Preoperative three-dimensional image constructed by Gamma Plan. (B) Surgeon's view via the left retrosigmoid approach clearly demonstrates the anatomical relations of the trigeminal nerve and adjacent veins (yellow: trigeminal nerve; blue: superior petrosal vein; green: pontomesencephalic vein).

There is no established treatment for AVM-related TGN. For patients with AVM-related TGN, treatment for both neuralgia and risk of hemorrhage from AVM should be taken into account. Besides open surgery, stereotactic radiosurgery and/or endovascular embolization of the nidus have been reported. ${ }^{3,7-9}$ Most of the reported cases showed good result in control of the TGN by reducing the flow of the nidus. As for open surgery, the procedure would differ depending on the cause of TGN. In case of a small AVM on the intrinsic trigeminal nerve, total resection of the nidus would be the ideal treatment as decompression of the nerve and elimination of potential risk of hemorrhage from the AVM can be achieved simultaneously. ${ }^{4}$ On the other hand, some authors reported good result only by decompression of the trigeminal nerve without excision of the nidus. ${ }^{1}$ Complete removal is not always easy in some cases. Furthermore, the risk of the hemorrhage from incidental AVM is not well known yet. Therefore, it is difficult to determine the optimal treatment for AVM-related TGN.

Our case is similar to the one reported by Sato et al, ${ }^{10}$ except for the location of the nidus. The trigeminal nerve was compressed by lateral and medial pontomesencephalic veins. They performed interposition by inserting Teflon felt at root entry zone 1 year after failed radiosurgery, resulting in good outcome immediately after the operation. In our case, we estimated that flow reduction by radiosurgery or embolization would not be practical as the nidus was too large and considered MVD as the treatment of choice for the patient's intolerable pain. It was found impossible to perform complete transposition because the venous wall was very fragile and it might be difficult to manage hemorrhage from the red veins. We used Teflon felt for interposition. Although the complete pain relief was achieved and the pain has not recurred yet 6 months after the surgery, we consider the risk of the recurrence would be relatively high as interposition technique was the only available procedure in this case. Long-term follow-up and accumulation of the cases of AVM-related TGN is necessary to establish optimal treatment for this rare TGN.

\section{Conclusion}

A rare case of TGN caused by draining veins from supratentorial AVM is reported. Interposition is safer than transposition as for MVD in this particular case.
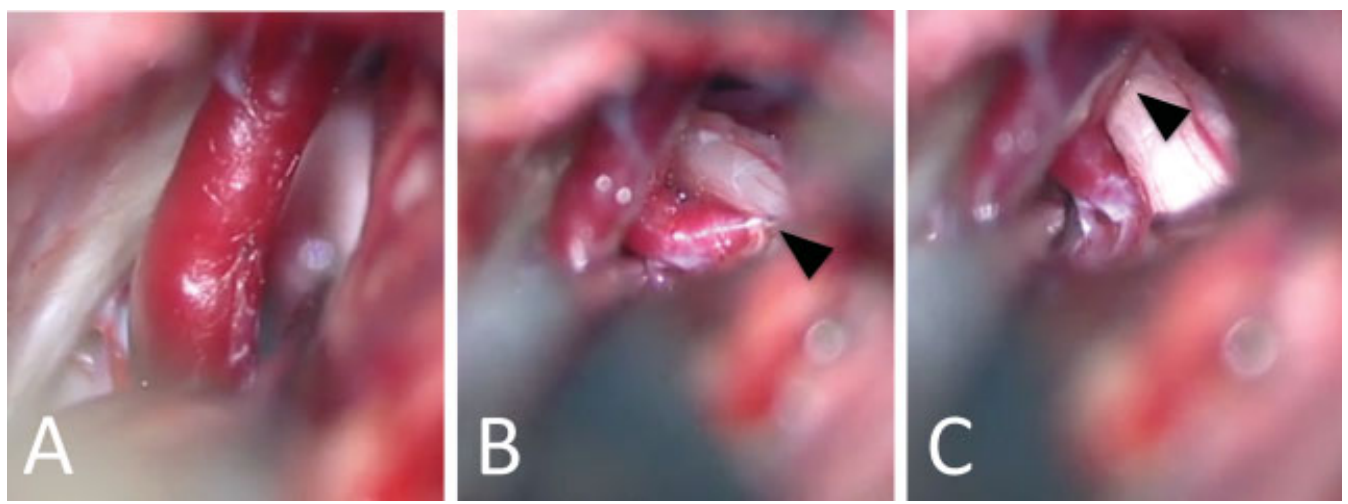

Fig. 4 Intraoperative photos. (A) Dilated and arterialized superior petrosal vein. (B) Trigeminal nerve is compressed by red vein at its root entry zone (black arrowhead). (C) The distal portion of the nerve is compressed by the red vein behind the suprameatal tubercle (black arrowhead). 
e112 Trigeminal Neuralgia Due to an AVM Draining Vein Inoue et al.

\section{References}

1 García-Pastor C, López-González F, Revuelta R, Nathal E. Trigeminal neuralgia secondary to arteriovenous malformations of the posterior fossa. Surg Neurol 2006;66(2):207-211, discussion 211

2 Karibe H, Shirane R, Jokura H, Yoshimoto T. Intrinsic arteriovenous malformation of the trigeminal nerve in a patient with trigeminal neuralgia: case report. Neurosurgery 2004;55(6):1433

3 Mori Y, Kobayashi T, Miyachi S, Hashizume C, Tsugawa T, Shibamoto Y. Trigeminal neuralgia caused by nerve compression by dilated superior cerebellar artery associated with cerebellar arteriovenous malformation: case report. Neurol Med Chir (Tokyo) 2014;54(3):236-241

4 Edwards RJ, Clarke Y, Renowden SA, Coakham HB. Trigeminal neuralgia caused by microarteriovenous malformations of the trigeminal nerve root entry zone: symptomatic relief following complete excision of the lesion with nerve root preservation. J Neurosurg 2002;97(4):874-880
5 Apfelbaum RI. Surgery for tic douloureux. Clin Neurosurg 1983; 31:351-368

6 Ishikawa M, Nishi S, Aoki T, et al. Operative findings in cases of trigeminal neuralgia without vascular compression: proposal of a different mechanism. J Clin Neurosci 2002;9(2):200-204

7 Anderson WS, Wang PP, Rigamonti D. Case of microarteriovenous malformation-induced trigeminal neuralgia treated with radiosurgery. J Headache Pain 2006;7(4):217-221

8 Lesley WS. Resolution of trigeminal neuralgia following cerebellar AVM embolization with Onyx. Cephalalgia 2009;29(9):980-985

9 Simon SD, Yao TL, Rosenbaum BP, Reig A, Mericle RA. Resolution of trigeminal neuralgia after palliative embolization of a cerebellopontine angle arteriovenous malformation. Cent Eur Neurosurg 2009;70(3):161-163

10 Sato K, Jokura H, Shirane R, Akabane T, Karibe H, Yoshimoto T. Trigeminal neuralgia associated with contralateral cerebellar arteriovenous malformation. Case illustration. J Neurosurg 2003;98(6):1318 